\title{
Editorial: Urban Computing in Mobile Environment
}

\author{
Xiaoxian Yang ${ }^{1} \cdot$ Ying $\mathrm{Li}^{2}$ \\ Published online: 11 May 2020 \\ (C) Springer Science+Business Media, LLC, part of Springer Nature 2020
}

\section{Editorial comment:}

With the acceleration of urbanization and the rapid development of the Internet, mobile devices have become an indispensable element in daily life for most people. A large volume of data has been generated by a diversity of sources in urban spaces, such as mobile App, sensors, vehicles, buildings, and human beings. If these produced data are improperly stored, published, or processed, it would lead to a waste of data resources. It would also lead to privacy disclosure, and attackers could take such an opportunity to steal privacy from the leaked privacy. On the contrary, if these data can be stored, published and analyzed appropriately, it can protect privacy hidden in the data, and can also tackle issues that many cities are facing, such as air pollution, traffic congestion, and urban planning. To acquire deep insight into these data, people develop urban computing, which contains a series of techniques, including data management, data mining, and prediction analysis. Urban computing aims to help urban managers to improve the quality of urban planning and construction, as well as to solve various issues and emergencies in urban development. This special issue includes six selected papers with high quality.

The first article titled Device-free Indoor Multi-target Tracking in Mobile Environment proposes DCT (a noise-tolerant, unobtrusive, and device-free tracking framework) to explore only to use RSS (Received signal strength) measurements for the fast multiple targets tracking. The authors propose a density-based clustering method to measure the number of targets. They also adopt a linear function based on empirical measurements to refine the number of targets, and then further use the FCM algorithm to modify the target

Xiaoxian Yang

xxyang@ @spu.edu.cn

1 School of Computer and Information Engineering, Shanghai Polytechnic University, Shanghai, China

2 College of Computer Science and Technology, Zhejiang University, Hangzhou, China

locations. Based on multiple particle filter (MPF), the trajectory of targets is finally obtained.

The second article titled A Topic Attention Mechanism and Factorization Machines based Mobile Application Recommendation Method presents a BiLSTM model with an enhanced topic attention mechanism, which is applied to mobile application recommendation and achieves good results. This model incorporates the topic information of mobile application description text into BiLSTM structure with attention mechanism through the LSA model and obtains the mobile application text representation vector that contains rich local semantic information and strong global topic information. A full connection layer is used to get the probability distribution of the text vectors belonging to different categories by using the softmax activation function, for the classification of mobile applications.

The third article titled A Novel Approach to Scheduling Workflows Upon Cloud Resources with Fluctuating Performance considers that the data privacy of mobile users and the response speed of data processing in cloud platforms are hard to guarantee due to the massive perception data in participatory MCS system. The authors propose a three-layer mobile crowdsensing system structure, mainly crowds, edge servers, and cloud platforms, and introduce edge servers to improve the response speed and ensure high reliability of services. Then they study the social welfare maximization problem based on the market economy model and Markov decision process. Based on that, a pricing approach is proposed which includes two kinds of incentive mechanisms to maximize social welfare.

The fourth article titled Mining Consuming Behaviors with Temporal Evolution for Personalized Recommendation in Mobile Marketing Apps focuses on providing a personalized and accurate recommendation in mobile marketing. First, the authors mine the periodic trends of users' consuming behavior from historical records by $\mathrm{KNN}$ (K-nearest neighbor) and SVR(support vector regression) based time series prediction, and predict the next time when a user re-purchases the item so that they can recommend the items. Second, to find the regularity of users' purchasing behavior during different life stages 
and recommend the new items, they mine the mapping model from items to the user's life stage first. Based on the model, users' current life stage can be estimated from their recent behaviors.

The fifth article titled JUSense: A Unified Framework for Participatory based Urban Sensing System points that participatory sensing has brought a new paradigm to smart city applications. The authors have proposed a unified framework named JUSense (Judicious Urban Sensing) with its core based on participatory sensing. JUSense combines and integrates four major independent subsystems namely AirSense, NoiseSense, SenseDCity, and D\&RSense to address societal issues like air pollution, noise pollution, aggressive driving, poor road condition, etc. JUSense provides the opportunity for applications to tackle the challenges in the steps of data collection, aggregation of data in the cloud, calibration, data cleaning, and prediction.

The sixth article titled Quantifying Memories: Mapping Urban Perception explores how spatial comprehension is influenced by the pattern of the spatial layout in urban settings and by individuals' daily activities. The authors create a web-based visual survey in the form of a geoguessing game. The participants were asked to guess the locations of random street views within a familiar neighborhood by placing a pin on a map. This system can measure how well they remember different urban images based on two indicators of spatial familiarity: location identification and visual recognition. By analyzing the results, they can clarify previously unknown aspects of the cognitive role in exploring the built environment and on hidden patterns embedded in the relationship between the city's spatial elements and people's mental maps.

Acknowledgments The guest editors are thankful to our reviewers for their effort in reviewing the manuscripts. We also thank the Edit-in-Chief, Dr. Imrich Chlamtac, for his supportive guidance during the entire process. We also thank you for the support from the National Natural Science Foundation of China (NSFC) under Grant No. 61902236.

Publisher's note Springer Nature remains neutral with regard to jurisdictional claims in published maps and institutional affiliations.

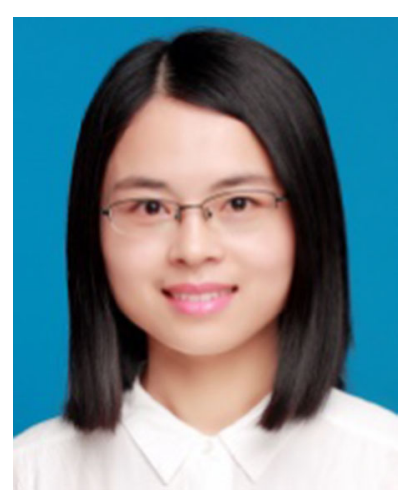

Dr. Xiaoxian Yang received the Ph.D. degree in Management Science and Engineering from Shanghai University, China, in 2017. She is currently an assistant professor at Shanghai Polytechnic University, China. Dr. Yang's research interests include business process management, formal verification, wireless network, and mobile health. Her researches are supported by the National Natural Science Foundation of China (NSFC), Natural Science Foundation of Shanghai (NSFS), CERNET Innovation Project, and Foundation of Science and Technology Commission of Shanghai Municipality. She has published more than 20 papers in academic journals such as TITS, TOMM, TCSS, TOIT, FGCS, MONET, IJSEKE, FGCS, IJDSN, Remote Sensing, and international conferences such as CollaborateCom, SEKE and IDEAL. She obtained 2 patents and 3 registered software copyrights in China, involving Wireless Network, Workflow Management, and Formal Verification. Dr. Yang had participated in organizing international conferences and workshops, such as CollaborateCom 2018, Chinacom 2019 and Mobicase 2019. She also worked as Guest Editor for MONET and WINE, and served as reviewers for IEEE TII, Wiley ETT, FGCS, PPNA, JBHI, Wireless Networks, COMPUTER NETWORK, etc.

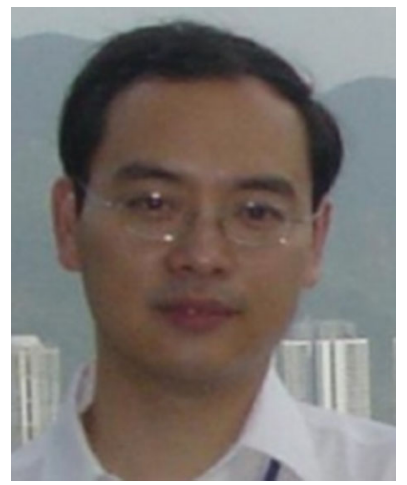

Prof. Ying $\mathbf{L i}$ is an associate professor in College of Computer Science and Technology, Zhejiang University, director of the Center for Pension Health Innovation Service Research, Binhai Industry Technology Research Institute of Zhejiang University. In 2000, he received his $\mathrm{Ph} . \mathrm{D}$. degree in computer software from Zhejiang University. $\mathrm{He}$ worked as a visiting scholar at the University of California, Santa Barbara (UCSB) in 2013. $\mathrm{He}$ was selected into the talent project in Zhejiang New Century 151 . He has been engaged in research in the fields of service computing, platform software, business process management and other industrial applications. Dr. Li has undertaken more than 10 scientific research projects as the leader, including 6 national-level projects such as the National Major Special Project, the National Science and Technology Support Program, the National 863 Program and the National Natural Science Foundation Project. The research and development of the Qiantang middleware platform software and other achievements won the second prize of the National Science and Technology Progress Award and the first prize of the provincial and ministerial level scientific and technological progress. More than 50 research papers have been published, of which more than 30 papers have been indexed by SCI/EI. More than 60 national invention patents were proposed. He focused on the research on common support technologies in the field of modern service industry and had invented a series of core technologies around complex service systems. 